\title{
Research Progress and Advantage Analysis of Big Data Application in Chinese Academic Journals
}

\author{
Qin WU $\mathrm{W}^{\mathrm{a}, \mathrm{b}, 1}$ and Hui WU ${ }^{\mathrm{c}}$ \\ ${ }^{a}$ Editorial Department of Journal, Shandong Jianzhu University, Jinan, China \\ ${ }^{\mathrm{b}}$ School of Art, Shandong Jianzhu University, Jinan, China \\ ${ }^{\mathrm{c}}$ Longquan Primary School in Tai'an high-tech Industrial Development Zone, \\ Tai'an, China
}

\begin{abstract}
The mining and application of big data in academic journals can accelerate the construction of data journals, enhance journal's influence, and promote the sharing and dissemination of scientific data in academic journals worldwide. This paper uses bibliometric method to retrieve published articles with the theme of big data and journal in CNKI database, analyzes the academic achievements of the development of academic journals with the application of big data in the recent five years using quantitative visualization analysis, expounds the research progress of academic journals in big data field, and discusses the advantages of big data application in periodical industry. The results show that: study on the application of big data in academic journals are gradually deepening and scientific, and the relevant research still needs more financial fund from the state and social units, big data has prominent advantages such as accuracy, scientificity and value maximization in the workflows of academic journals. The mining and application of massive data is very important for promoting the development of high-quality academic journals and optimizing the supply-demand relationship of knowledge services of academic journals.
\end{abstract}

Keywords. big data, academic journals, bibliometric method, advantages analysis

\section{Introduction}

Big data is not simply a huge amount of data, but a data collection that greatly exceeds the capabilities of traditional database software tools. It has four characteristics: massive data scale, fast data flow, diverse data types and low value density [1]. The feature of big data lies in the distributed data mining, analysis and processing of massive data, so that people can make more scientific and well founded decisions. As another disruptive technological revolution after cloud computing and internet of things, big data has been widespread used in various research fields of natural science and social science, providing a new research paradigm and quantitative method for various disciplines [2]. The effective application of big data will be the core of the common

${ }^{1}$ Qin Wu, Editorial Department of Journal/School of Art, Shandong Jianzhu University, Jinan 250101 China; E-mail:1063983400@qq.com 
competition of all industries. The mining and application of massive data is entering and deeply affecting every industry, organization and unit.

Academic journals are the windows to reflect the level of scientific and technical innovation of a country, and the sci-tech carrier to spread new discoveries and new knowledge [3]. In the process of knowledge dissemination and service, it is necessary for academic journals to make full use of big data to mine its potential value [4]. As an organic part of the national independent innovation system, academic journals are closely related to scientific research and play an important role in sci-tech support. Big data is both a challenge and an opportunity for the development of Chinese academic journals [5]. However, at present, the general progress of the research on the relationship between the development of academic journals and big data is not clear, resulting in a lack of profound grasp of the application and necessity of big data in journal industry, under the background of the fast development of big data, academic journals, such as some university journals and local trade sci-tech journals, are also faced with the problems of limited data resources, lower accuracy of information, and lower value of data reuse. Therefore, the establishment of organic integration and effective collaboration between academic journals and big data technology is a problem in need of immediate solutions in the information dissemination of academic journals in big data era.

This paper uses bibliometric method to retrieve published articles with the theme of big data and journal in China national knowledge infrastructure (CNKI) database, analyzes the retrieved documents through quantitative visualization analysis, so as to understand the research situation of big data application in Chinese academic journals, illustrates the application advantages of big data in academic journals. It is helpful to promote the efficient application of big data and the technology, optimize the allocation of academic journal resources, improve the accuracy, scientificity and value-added of journal publishing process, and enhance the frontier and creativity of knowledge and information dissemination of Chinese academic journals. This study may have theoretical guidance and reference significance for the related School of Art as well as primary school education.

\section{Research progress of big data and the development of academic journals}

\subsection{Data sources}

All data in this paper are from CNKI database.

\subsection{Research methods}

Log in to CNKI database and use advanced search functions to retrieve published articles with the theme of big data and journal. The time of publication is limited to January 1, 2015 to December 31, 2019. The retrieval time in this study is July 30, 2020. The retrieved literature is preliminarily screened, and some non-paper literature is removed, and the effective literature related to the subject is retained and analyzed visually; the retrieved data is imported into Excel to be analyzed. 


\section{Results and analysis}

\subsection{Numbers of published articles}

A total of 189 relevant literatures were retrieved in CNKI database from 2015 to 2019. 186 effective literatures related to the theme were retained after removing some nonpaper literatures. The numbers of published articles from 2015-2019 is shown in Figure 1. As can be seen from Figure 1, published articles about journal big data in recent five years showed a trend of first increasing and then decreasing. The year with the most published articles is 2016, with 53 articles, which is probably related to the high popularity of big data research and the rapid increase of attention in the periodical industry. From the data of the past three years from 2017 to 2019, the numbers of published articles shows a small decline, which may be due to the more rational and indepth research on big data in periodical industry, which leads to the reduction of literature output. It is believed that with the in-depth study of big data research in various industries, the promotion and application of big data will lead to a new round of research boom in the quality improvement, communication mode, digital upgrading and personalized service of academic journals.

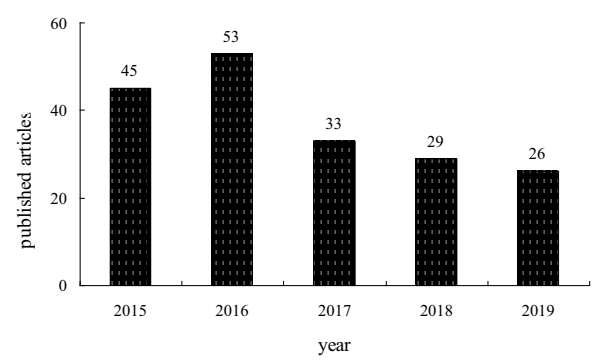

Figure 1.The numbers of published articles with the theme of big data and journal from 2015 to 2019

In 2020, The higher frequency of keyword co-occur network of articles on big data research is data technology and artificial intelligence in academic journals, which indicates that big data research has penetrated into all work links of academic journals [6-7], and has played a great role in promoting their brand building [8].

\subsection{Resource type distribution}

The academic literatures retrieved in this study are mainly periodical articles, accounting for $88.2 \%$, then of academic articles in newspapers, accounting for $4.8 \%$. The distribution of other resource types is shown in Figure 2. As can be seen from Figure 2, articles about journal big data research published in periodicals are the main resource type, indicating that the journal industry is closely related to big data research. Among the periodicals articles cited more frequently, Xia dengwu reconstructed the effective way of data cognition and value reconstruction of academic journals from the perspective of data [9], Wu Meiying's research show that big data research in academic 
journals has changed from exploring the causal relationship between the data to revealing its correlation [10].

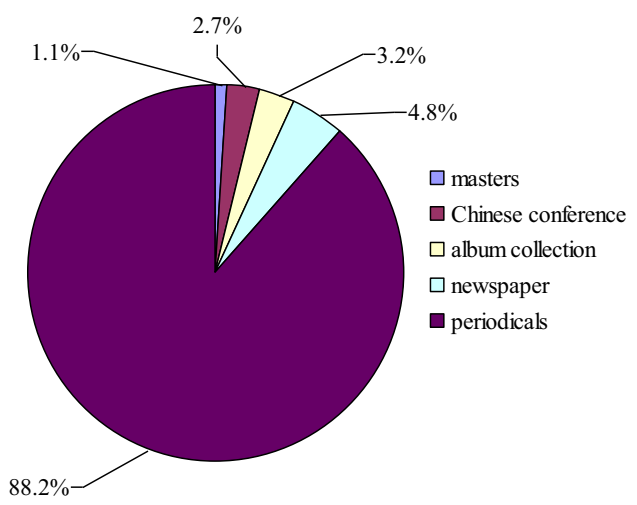

Figure 2. Distribution of resource types of published articles

\subsection{Fund distribution}

In fund distribution, published articles about journal big data research received less funding; only $3.8 \%$ of them were funded by the National Social Science Foundation, followed by educational research projects, accounting for 3.3\%. Other fund distribution is shown in Figure 3. It shows that more financial fund from the state and relevant departments is needed to support the research of journal big data.
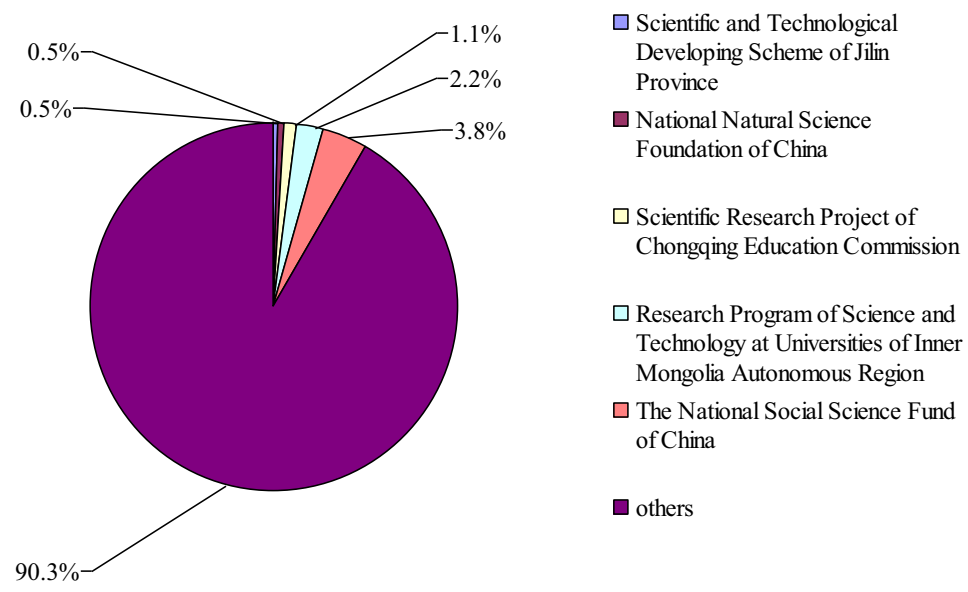

口 others

Figure 3. Fund distribution of published articles 


\section{Advantage analysis of big data application in academic journals}

With the coming big data era, the living environment of academic journals has undergone essential changes. Although the printed journals have certain vitality based on their authority, reliability and reality accumulated over the years, but in the era of big data, compared with the characteristics of printed academic journals, data journals have unprecedented huge advantages. These advantages are mostly reflected in the following aspects.

Accuracy. In big data era, knowledge and information are in a state of data. From the macroscopic view, by collecting and statistical analysis the academic data, academic journals can fully utilize the information provided by big data, grasp the academic development direction and scientific hot spot to enhance their influence [11]; from the micro view, using big data analysis to predict the audience group, academic journals can understand the personalized needs of authors and readers, and realize the intelligent matching between the knowledge service of academic journals and the personalized needs of the audience [12]. With the support of big data analysis, academic journals can timely obtain information feedback from users, accurately evaluate the dissemination effect of academic information, guide the direction of journal content integration, and realize the accurate positioning of academic guidance and knowledge service.

Scientificity. This advantage is mainly reflected in the evaluation function and mechanism of academic journals. At present, most comprehensive academic journals adopt the qualitative evaluation method of peer review, but this evaluation method is subjective. Big data analysis and application can provide an objective quantitative standard for this qualitative evaluation. On the basis of peer review, the influence and innovation of journals are quantified, and the combination of qualitative evaluation and quantitative evaluation ensures the evaluation index and evaluation method of academic journals more scientific [13-14].

Value maximization. In big data era, journal industry and data technology are deeply integrated. Through the cooperation with network database, academic journals deeply mine the knowledge information and academic resources using the big data analysis ability of open data platform, and excavate valuable data for future trends and predictive pattern analysis of academic journals [15]. Through big data analysis, the value of information resources of academic journal can be deeply excavated to produce more derivatives, and to maximize the value of knowledge services of academic journals.

\section{Conclusion and discussion}

This paper uses bibliometric method to retrieve published articles with the theme of big data and journal in CNKI database, and analyzes the retrieved literature through quantitative visualization analysis. The results show that the researches on the development of academic journals and the application of big data are more in-depth and scientific, but such researches lack the support of scientific research funds from the state and social units, which is consistent with that of Guo Yi's result [16]. In the future, the funding for big data research in academic journals should be increased. Big data has outstanding advantages in the accuracy, scientificity and value maximization of academic journals. 
The data age creates more opportunities and challenges for periodical industry. Relying on big data and intelligent terminal technology platform, academic journals always adhere to high-quality content construction, enhance the core competitiveness, and strengthen the authority, reliability and credibility of journal content in big data era. The mining and application of massive data is of great significance in accelerating the prosperity of high-quality academic journals and optimizing the supply-demand relationship of knowledge services of academic journals, so as to better serve the progress of national science and economic.

\section{Acknowledgement}

This work was funded by Social Science Innovation and Development Research Project of Shandong Province (No.2019SSD014 )

\section{References}

[1] Chen ZQ .The necessity of journals using big data. Journalism Tide. 2020 Jan; 1:62-64.

[2] Wu HJ, Sun HF, Sun T, Tian C. Frontier evolution of international BigData in knowledge cluster perspective. Information Science. 2019 May; 37(5):173-177.

[3] Yan XM .The path to improve the communication power of sci-tech journals in the era of big data. China Publishing Journal. 2019 Jun; 12:64-65.

[4] Huang F. Research on operation practice of WeChat public account and big data of university science and technology journal. Communication and Copyright. 2019 Oct; 8:103-107.

[5] Guan LL, Ma HQ, Wang CL. Practice and thoughts on establishing international data journals: A case study of Big Earth Data. Chinese Journal of Scientific and Technical Periodicals. 2020 Jan; 31(1):56-62.

[6] Tan XJ. Application of big data and artificial intelligence in editing of comprehensive academic journals. Public Communication of Science and Technology. 2020 Mar; 3:11-13.

[7] Jiang XD, Tu P, Yin LX. Topic selection planning of scientific journals from the perspective of data mining and intelligent screening. Publishing Journal. 2020 Jan; 28(1):36-41.

[8] Yang YJ. The role of big data technology in promoting the brand building of academic journals. Media Forum. $2020 \mathrm{Jul} ; 3(17): 85-86$.

[9] Xia DW. Content optimization and value reconstruction of academic journals in the data era. Chinese Journal of Scientific and Technical Periodicals. 2016 Mar; 27(3):264-268.

[10] Wu MY. Academic journal editors'quality reconstruction and capacity improvement in the era of big data. Publishing Journal. 2017 Jan; 25(1):47-51.

[11] Zhou J. Thought and practice on optimizing publishing process of scientific journals by big data. Chinese Journal of Scientific and Technical Periodicals. 2018 Feb; 29(2):144-147.

[12] Zhou X, Qin Q. Knowledge management innovation and international influence promotion of academic journals under the background of big data.View on Publishing. 2018 Apr; 5:12-15.

[13] Zhao Y, Kong Q. Statistics and analysis of evaluation indexes of Chinese science and technology periodicalsin the big data era. Journal of Qingdao University(Engineering \& Technology Edition). 2019 Nov; 34(4):98-102.

[14] Jiao B. Comprehensive academic journals under the background of big data.Chinese Social Sciences today. 2016-07-12. http://www.cssn.cn/zx/201607/t20160712_3117419.shtml

[15] Wu H, Chen XF, Wang F, Huang JX. Study on global OA journal publishing monitoring method. Journal of Modern Information. 2019 Feb; 39(2):145-151.

[16] Guo Y, Chi L, He XG. Overview of big data and the development of Chinese sci-tech journal. Development and Orientation of Sci-tech Periodicals. 2018 Jul; 11:45-50. 